\title{
Giant magnetoresistance
}

\section{Stephen Ornes}

Science Writer

Scientists have known for more than 150 years that a magnetic field can change electrical resistance in a material, ever since Scottish physicist William Thomson (Lord Kelvin) first measured the effect in iron. That phenomenon, magnetoresistance, can be tweaked according to the strength and orientation of the field, and has made it possible for engineers to build sensors that could read the data stored as magnetic bits in the hard drives of early computers.

However, as digital devices became smaller their hard drives became more crowded. The trend toward miniaturization might have stalled in the 1990s if it weren't for the advent of a new technology that powered the design and implementation of more

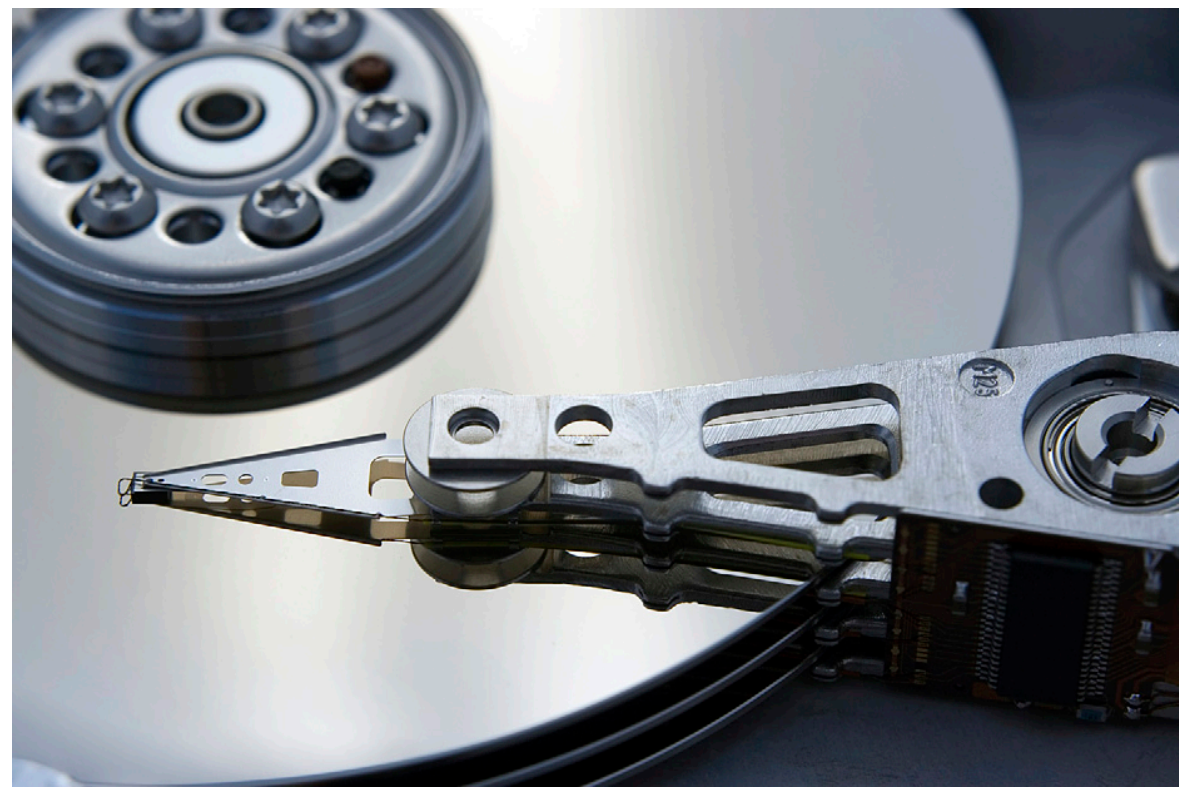

A read/write head sits above a computer hard disk, manipulating the stored information by the use of giant magnetoresistance.@iStockphoto.com/BrandonSeidel laptops, cell phones, search engines, and music players to scour densely packed hard drives and extract data in less time than it takes to push a button.

Two European physicists, Albert Fert from France and Peter Grünberg from Germany, independently demonstrated GMR in custom-built devices in 1988, and in 2007 the two shared the Nobel Prize in Physics10 years after the first GMR-based read-out head showed up in a digital device.

Both scientists built devices by alternating layers of magnetic and nonmagnetic materials, each layer only atoms thick, in a kind of nanosandwich. Although their initial prototypes worked only at supercold temperatures, other physicists soon invented the materials and methods to bring the technology to room temperature.

Read-out heads that use GMR have the same basic design as Fert's and Grünberg's originals. In a read-out head that uses GMR, the separated magnetic layers are magnetized in opposite directions. An electric current moves through the magnetic-nonmagneticnanosandwich. However, because the intrinsic magnetic properties of the electron (its spin) and one of the layer's magnetic fields point in different directions, the electrons in the current encounter high resistance.

Enter the spinning hard drive: As the outermost magnetic layer of the read-out head moves over a magnetic bit in the spinning disk, this layer's magnetic field aligns with that of the bit and of the other magnetic layer. As a result, the resistance drops off dramatically and the electrons in the current sail through. This dramatic change in resistance shows the binary state of the bit. Because GMR is so sensitive to even slight magnetic perturbations, bits can be supersmall and, therefore, packed more tightly on a disk.

Although GMR now shows up in the memory-readers of digital devices, engineers are exploring ways to use it for all kinds of memory, including random-access memory, or RAM. 